\section{The jet set}

Mario Livio

A lthough a wide variety of astrophysical objects produce powerful jets, we still lack a comprehensive theory of their formation. In our Galaxy, young stellar objects, massive X-ray binaries, black hole $\mathrm{X}$-ray transients, symbiotic stars, supersoft $\mathrm{X}$-ray sources and even some planetary nebulae are all accreting systems that produce jets. Among extragalactic sources, powerful jets are observed in active galactic nuclei (accreting, supermassive black holes) and are thought to exist in $\gamma$-ray bursts (which possibly involve accreting, stellar-mass black holes).

Despite the ubiquity of jets, there is no generally accepted theory for the mechanism of their acceleration and alignment. Early attempts to explain this collimation involved nozzles (similar to rocket exhausts), in which an adiabatic flow propagating in a medium with decreasing pressure first converges and then diverges as it accelerates to supersonic speed. Another idea was that the collimating agents are funnels that are formed at the centres of dense tori. Radiation pressure on electron-positron pairs, on resonance lines,

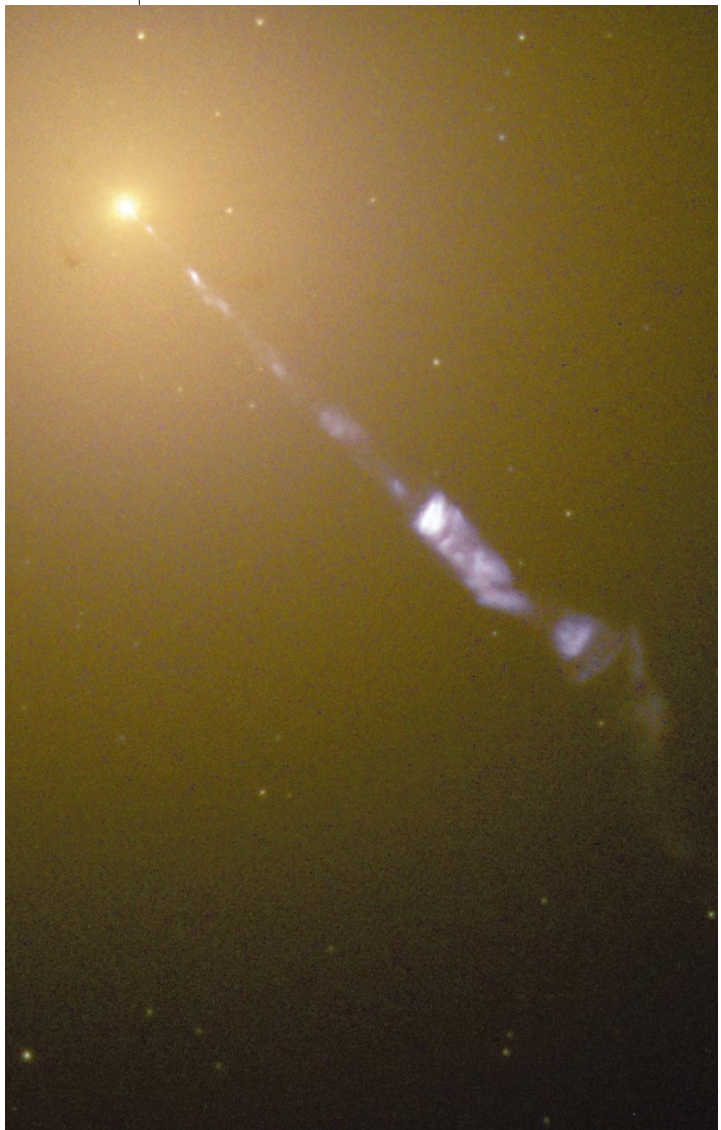

Shining example: the M87 galaxy spews out its jet. or on dust, have been suggested to accelerate jets in some of the systems mentioned above.

The main problem of these early models is that they do not work for all classes of astrophysical jets. For example, although the power emitted by the central source approaches the critical Eddington luminosity (at which radiation pressure exactly balances gravity) in supersoft X-ray sources, this is not true for most other classes of objects. The drag caused by radiation limits the attainable speeds to values below those observed in other (ultrarelativistic) jets. Similarly, dense tori with narrow axisymmetric funnels, which were once assumed to exist around black holes, were later shown to be unstable to non-axisymmetric instabilities that could destroy them on a dynamic timescale. Can a universal mechanism of acceleration and collimation that operates in all classes be found?

The signs are positive. First, there is strong observational evidence that almost all jet-producing systems contain an 'accretion disc' around a compact object. This disc is both a source of energy (in the compact object's gravitational potential) and provides the required axial symmetry. Second, jet velocity is always of the order of the escape velocity from the central object, indicating that jets originate from the centre of the accretion disc. The Galactic black hole transient source GRS 1915 + 105 (a microquasar) provides even more evidence for the connection between the jet and the central part of the disc, and we can begin to see a picture in which the inner disc is episodically accreted while ejecting a relativistic plasma, which subsequently produces infrared and radio flares by synchrotron emission.

The most promising universal mechanism for jet acceleration and collimation relies on an accretion disc threaded by a poloidal, large-scale magnetic field. The basic idea is that some magnetic flux is in open field lines, which form a certain angle with the disc's surface. Ionized material is forced to follow field lines; as these lines are anchored in the disc and rotate with it, material is centrifugally accelerated like a bead on a wire. The jet may be collimated by hoop stresses resulting from the magnetic field's toroidal component; alternatively, if the poloidal magnetic flux is largest at the disc's edge, and the disc's radius is large relative to that of its central object, the jet will be collimated by the magnetic field lines.

Some differences in behaviour remain unexplained by a unified mechanism. For example, jets from the nuclei of 'blazars' active galaxies from which jets are beamed in

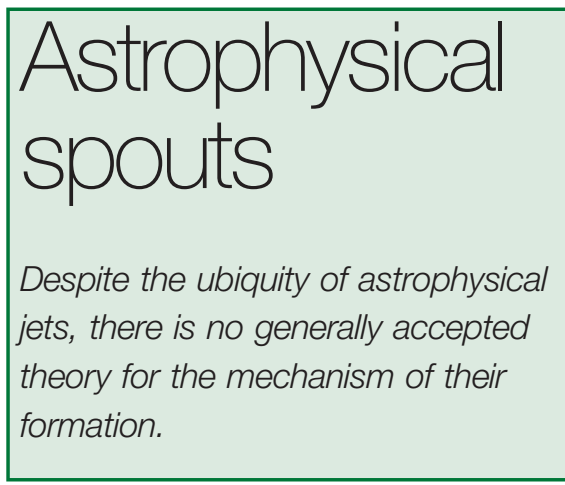

the direction of the observer - are ultrarelativistic, whereas jets from Seyfert galaxies are not. Yet in both cases, jets are thought to originate from the centres of discs around black holes. The difference may be due to different environments, different mass-loading of the magnetic field lines, or something else altogether.

Extraction of rotational energy from a rapidly spinning (Kerr) black hole by magnetic fields that permeate the hole's event horizon is often invoked as a means of launching jets in active galactic nuclei. The mechanism itself can be understood if the black hole is likened to a resistor rotating in a magnetic field and generating a potential difference between the hole's pole and equator. X-ray observations of the Seyfert galaxy MCG-6-30-15 and the Galactic black hole candidate XTE J1650500 have now revealed an extremely broad and redshifted iron $\mathrm{K} \alpha$ line, indicating an origin in the very centre of the accretion disc. This implied emissivity may mean that energy is indeed extracted from the spinning black hole. If true, this could show that an exotic process anchored in electromagnetism in curved spacetime is operating across a range of a factor of a million in black-hole mass!

This interpretation, however, is far from unique. Much better understanding of processes within the plunging orbits between the innermost stable orbit in the accretion disc and the black hole's event horizon will be needed to draw a final conclusion.

Mario Livio is at the Space Telescope Science Institute, 3700 San Martin Drive, Baltimore, Maryland 21218, USA.

\section{FURTHER READING}

Mirabel, I. F. \& Rodriguez, L. F. Nature 392, 673-676 (1998).

Wilms, J. et al. Mon. Notices R. Astron. Soc. 328, L27-L31 (2001).

Celotti, A. \& Blandford, R. D. in Black Holes in Binaries and Galactic Nuclei: Diagnostics, Demography and Formation (eds Kaper, L. et al.) 206-215 (Springer, Berlin, 2001).

Livio, M. in Probing the Physics of Active Galactic Nuclei by Multiwavelength Monitoring (eds Peterson, B. M. et al.) 225-248 (ASP, San Francisco, 2001). 